\title{
A Model for Estimation of Noise Tolerance in ECG Parameters
}

\author{
Reza Firoozabadi, Richard E Gregg, Saeed Babaeizadeh \\ Advanced Algorithm Research Center, Philips Healthcare, Andover, MA, USA
}

\begin{abstract}
Parameters extracted from ECG recordings show different noise tolerance levels. Some parameters may be slightly affected by noise, while the others could be inaccurate. Choosing a single noise threshold for all parameters may lead to adoption of invalid results or removal of valid parameters. In this study, we develop a statistical model between the Signal-to-Noise Ratio (SNR) and our Signal Quality Indicator (SQI) algorithm, and determine the noise-tolerance threshold for several ECG parameters statistically. Our dataset was based on the STAFF-III database with added physical noise recording segments from MIT-BIH database containing electrode motion, muscle artifact and baseline wander. We generated 3193 noise-added 12-lead ECG signals with SNR varying from $-6 d B$ to $24 d B$. For each 10 -second segment, the noise level was measured by our SQI algorithm, and ECG parameters were measured by the Philips DXL ECG algorithm, allowing us to derive a noise model and the thresholds for noise tolerance of the ECG parameters. Varying thresholds suggest using parameterspecific thresholds in order to avoid reduction in accuracy.
\end{abstract}

\section{Introduction}

Some ECG parameters are fairly noise sensitive while the others may be essentially unaffected by high noise levels. Most clinical systems use a single noise tolerance threshold, overlooking the fact that the noise tolerance spans a wide range for different parameters. This may cause elimination of all measurements by the system although some measurements are still valid, or in the other extreme scenario it may lead to adoption of all measurements including the incorrect values. The inaccurate measurements could be misleading in clinical decision-making process if not marked. This emphasizes the importance of using a parameter-specific threshold.

By implementing an independent noise tolerance threshold for each parameter, we avoid treating all measurements in the same manner. In other words, the parameter-based noise tolerance threshold will help the operators avoid missing valid measurements or accepting incorrect ones and make the right decision whether a measurement should be used or ignored.

\section{Methods}

\subsection{Database}

We developed a controlled noise-added ECG database where 31 different SNR levels were created by adding noise to each ECG recording. A total of 3193 12-lead ECG recordings with SNR from -6dB to $24 \mathrm{~dB}$ were generated.

The base ECG recordings $(n=103)$ were chosen from the STAFF-III database [1] which is a set of recordings collected in patients undergoing prolonged percutaneous transluminal coronary angiography (PTCA) as a model to study ischemia using balloon inflation. The recordings contain a 5-minute pre-inflation ECG segment at rest in supine position before the catheter insertion, and several during and after balloon inflation. We used 5-minute prePTCA 12-lead ECG segments as the base ECG.

The noise recordings from MIT-BIH database [2] were selected as the additive noise. The database has three 2channel half-hour noise recordings typical in ambulatory ECG recordings. These recordings were generated using physically active volunteers and standard ECG equipment, by selecting the intervals containing predominantly baseline wander, muscle artifact, and electrode motion artifact. We combined the three noise types and split the noise recordings into six 5-minute two channels segments, an overall of 12 noise segments.

The 12-lead QRS peak to peak amplitudes (signal) and 12 noise segment RMS amplitudes were measured using the Physionet sigamp tool. Desired SNR was achieved by scaling the additive noise properly:

$$
\text { Noise-added ECG }=\text { Base ECG }+\alpha \text {.Noise }
$$

Signal to noise ratio (SNR) for the noise-added ECG is defined as:

$$
\begin{aligned}
& S N R=10 \log \frac{\mathrm{ppk}(\text { Base ECG })}{\alpha^{2} \cdot \mathrm{P}(\text { Noise })} \\
& \alpha=10^{(-S N R / 20)} \frac{\mathrm{ppk}(\text { Base ECG) })}{\sqrt{8} \cdot \mathrm{RMS}(\text { Noise })}
\end{aligned}
$$

Figure 1 shows an example of a 12-lead recording at $\mathrm{SNR}=0 \mathrm{~dB}$. Figure 2 illustrates lead II in the same example at various SNR levels. 


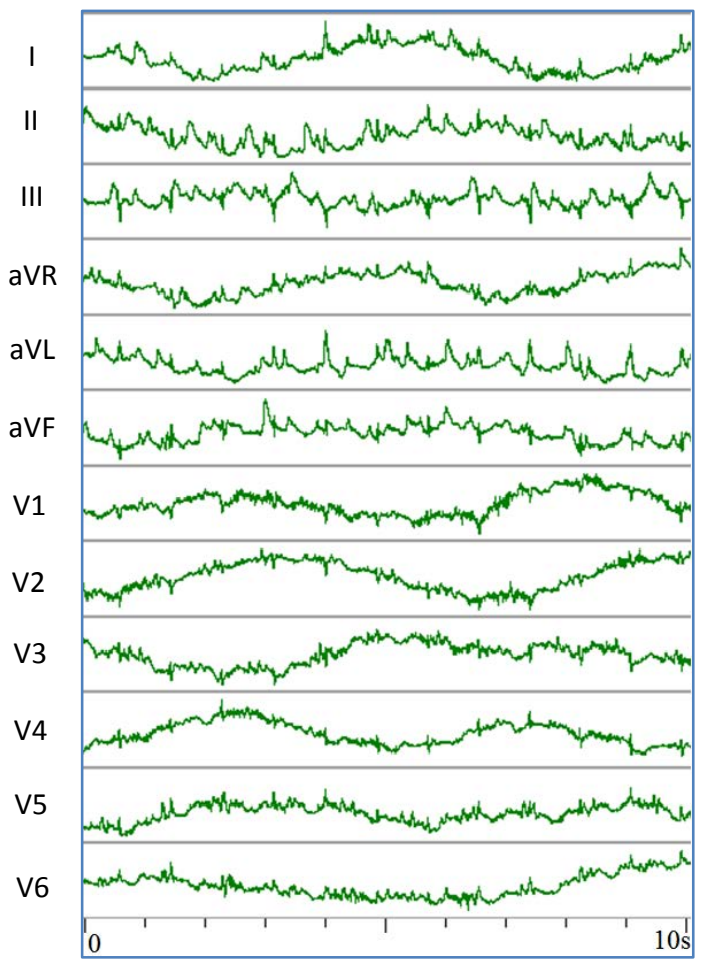

Figure 1. Example of a 12-lead noise-added recording at SNR of OdB.

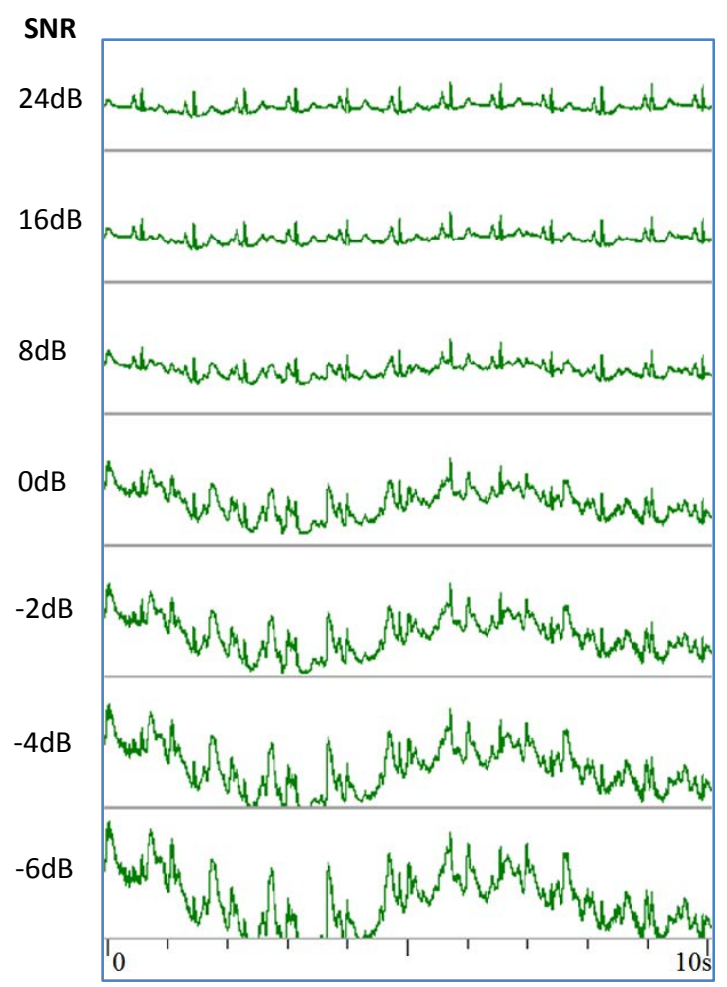

Figure 2. Example of lead II in a noise-added recording at various noise levels (signal to noise ratios at $24 \mathrm{~dB}, 16 \mathrm{~dB}$, $8 \mathrm{~dB}, 0 \mathrm{~dB},-2 \mathrm{~dB},-4 \mathrm{~dB}$, and $-6 \mathrm{~dB})$.

\subsection{Algorithm}

In a previous work [3], we presented our Signal Quality Indicator algorithm (SQI) and defined the noise level with a noise measure comprising high- and low-frequency components. In current work, we calculated the noise measure for each recording in our SNR-controlled database to develop a model mapping them to SNR values. Using Philips DXL ECG algorithm, Several ECG parameters were extracted from each 10-second noise added segment at different SNR levels. For each ECG parameter at any SNR, variation in values was observed and used as a measure of noise tolerance. The noise tolerance threshold for each ECG parameter was defined at the point the variations exceeded a pre-defined limit. The results are summarized in Table 2.

\subsubsection{Signal quality indicator}

As we described in [3], 10-second ECG recordings from the database are divided into 1-second 12-lead segments on which high- and low-frequency noise measures are calculated. The total noise measure in a 10-second recording is the combination of these two components averaged across all 1-second segments.

The high-frequency noise measure in a 1-sec segment is estimated as the median of the standard deviations of the ECG samples in the short-term low activity intervals described in [3]. The total high frequency noise measure in a 1-second segment $\left(N_{H F}\right)$ is the average of the high frequency noise measures across the leads.

The low-frequency noise measure is identified by the difference in the adjacent 1-second segment baselines which are calculated by averaging the ECG samples in the short-term low-activity intervals. It is the summation of differences between the current baseline and last 2 baselines. The total low-frequency noise measure in a 1second segment $\left(N_{B W}\right)$ is the average of the low-frequency noise measures across all leads.

Total noise measure is the weighted sum of the total high-frequency and low-frequency noise measures in each 1 -second segment, averaged across the 10-second racording:

$$
\text { Noise Measure }=\mathrm{E}\left[\alpha N_{H F}+\beta N_{B W}\right]
$$

The weighting between high frequency and low frequency noise measures is empirically based on perceived effect on total noise where the same level of high frequency noise has a much larger effect than baseline wander from beat to beat.

\subsubsection{Noise model}

To derive a model between the noise measures (NM) and SNR levels, we removed the effect of noise in the base 
ECGs by subtracting their noise measures from the calculated noise measures in each noise-added recording, then we normalized the result to an ECG with $1 \mathrm{mV}$ peakpeak amplitude. The median noise measures over all patients at each SNR were plotted in log scale and a log linear model was achieved by fitting a line:

$$
S N R=58.195-19.305 \log (N M)
$$

Figure 3 shows the log-linear model mapping the SNR levels to the normalized median noise measures.

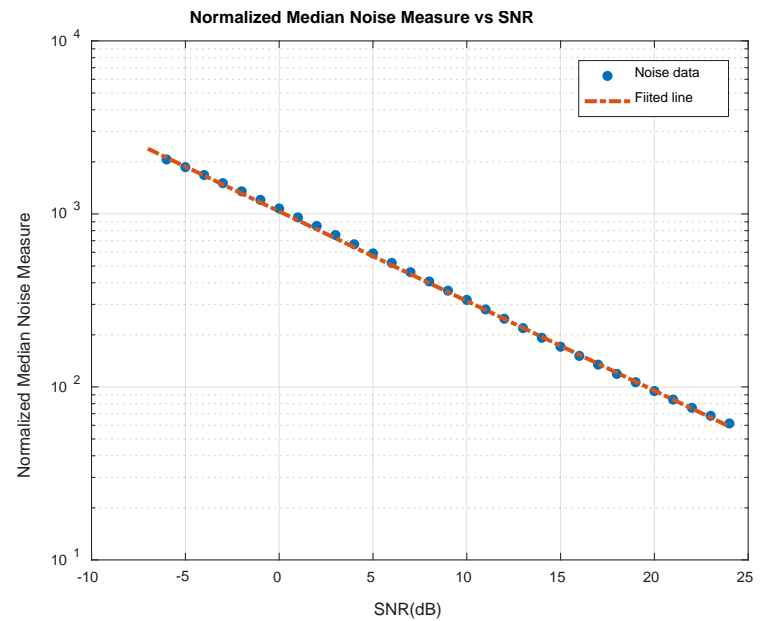

Figure 3. The log-linear model mapping the SNR levels to the normalized median noise measures. The dash-dot line was fitted to the normalized median noise measures displayed by dots.

\subsubsection{ECG Parameters}

The Philips DXL ECG algorithm [4] was run on every 10-second segment in each 5-minute noise-added recording. The algorithm measured several ECG parameters including those listed in Table 1.

Table 1. List of the ECG parameters and deviation limits

\begin{tabular}{ll}
\hline \hline ECG Parameter & Deviation limit \\
\hline \hline Heart Rate & $5 \mathrm{bpm}$ \\
PR interval & $15 \mathrm{msec}$ \\
QRS duration & $15 \mathrm{msec}$ \\
QT interval & $15 \mathrm{msec}$ \\
QT $_{\text {c interval }}$ & $15 \mathrm{msec}$ \\
P-wave axis & 10 degrees \\
QRS axis & 10 degrees \\
T-wave axis & 10 degrees \\
P-wave amplitude & $25 \mu \mathrm{V}$ \\
Q-wave amplitude & $25 \mu \mathrm{V}$ \\
R-wave amplitude & $100 \mu \mathrm{V}$ \\
S-wave amplitude & $25 \mu \mathrm{V}$ \\
T-wave amplitude & $25 \mu \mathrm{V}$ \\
ST amplitude & $25 \mu \mathrm{V}$ \\
ST-VM & $25 \mu \mathrm{V}$ \\
\hline
\end{tabular}

\subsubsection{Noise tolerance}

ECG parameters are measured by Philips DXL ECG algorithm every 10 seconds in a 5-minute noise-added recording. Higher variation in these values is a measure of the higher noise level. Figure 4 shows an example where the variation in ST-VM parameter (ST segment Frank lead vector magnitude) is increased by lowering the SNR.
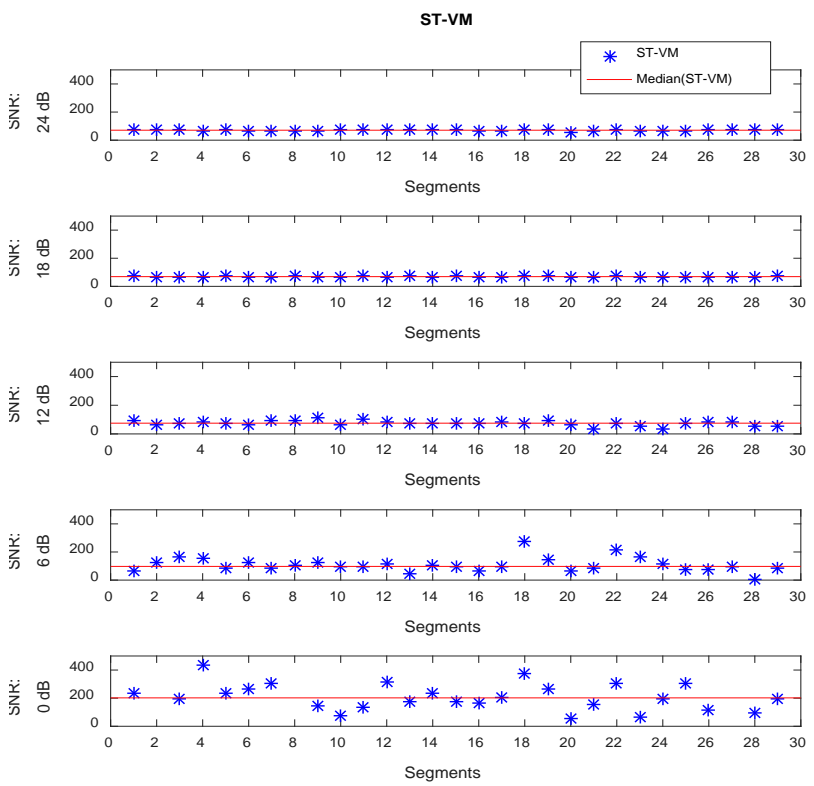

Figure 4. An example of higher variation in ECG parameter ST-VM by increasing the noise level.

The noise tolerance threshold for each ECH parameter in a patient recording is defined above the highest noise level where the deviation of the $1^{\text {st }}$ and $3^{\text {rd }}$ quartiles, as well as the base ECG median, from the parameter median at this noise level is still below a pre-defined deviation limit as shown in Table 1 . The noise tolerance threshold is defined in the middle point between the highest noise level meeting the following criteria and the next noise level:

$$
\mathrm{M}-\mathrm{Q}_{1}<\Delta \text { and } \mathrm{Q}_{3}-\mathrm{M}<\Delta \text { and }\left|\mathrm{M}-\mathrm{M}_{0}\right|<\Delta
$$

where $\mathrm{Q}_{1}, \mathrm{M}$, and $\mathrm{Q}_{3}$ are the ECG parameter values at $1^{\text {st }}$ quartile, median and $3^{\text {rd }}$ quartile of the data at the current noise level, $\mathrm{M}_{0}$ is the parameter median in the base ECG, and $\Delta$ is the deviation limit defined in Table 1.

Figure 5 shows an example where the variations in ST-VM parameter in a patient recording are measured at various noise levels. The errorbars show the values ranging between the $1^{\text {st }}\left(\mathrm{Q}_{1}\right)$ and $3^{\text {rd }}\left(\mathrm{Q}_{3}\right)$ quartile where the median is shown too. For this patient, the noise tolerance threshold is chosen at 651. At the next noise level, deviation of the parameter value at the $1^{\text {st }}$ quartile from the median exceeds the deviation limit of $25 \mu \mathrm{V}$. We observe an increase in the median values as the parameter is the magnitude of ST vector and is always positive. 


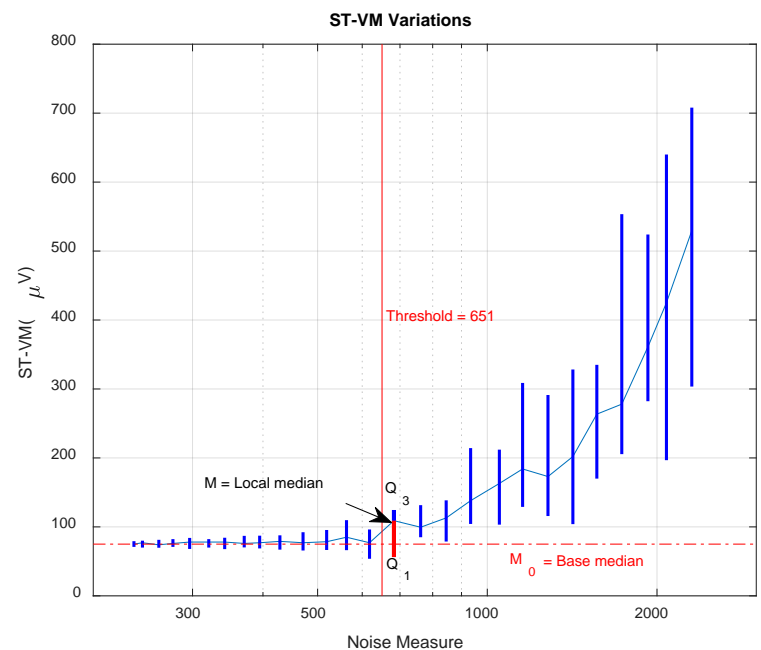

Figure 5. An example of variations in ST-VM parameter in a patient recording at various noise levels. The errorbars show the values ranging between the $1^{\text {st }}\left(\mathrm{Q}_{1}\right)$ and $3^{\text {rd }}$ $\left(\mathrm{Q}_{3}\right)$ quartiles. The median is also plotted. Threshold is chosen at 651 .

The total noise tolerance threshold for each parameter is defined as the median of thresholds for all patients. As an example, Figure 6 shows the histogram of noise tolerance thresholds for ST-VM parameter in all patient recordings with the median at 490 .

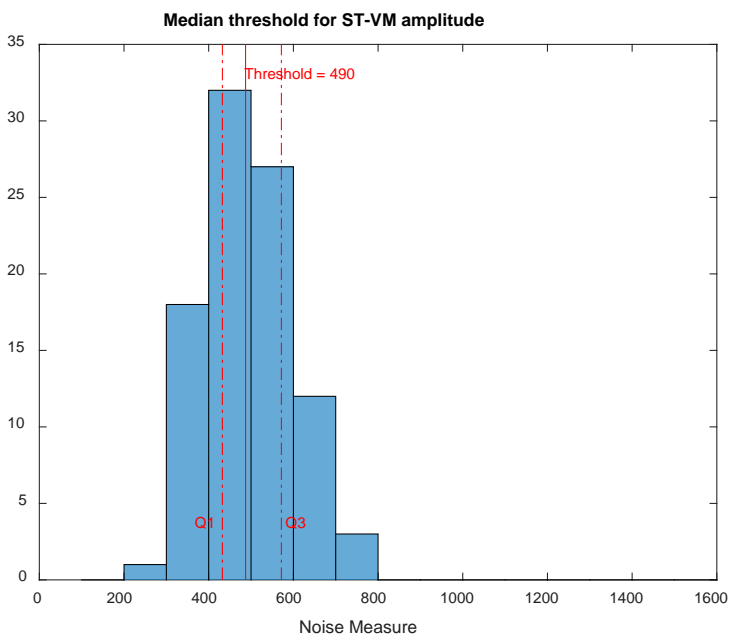

Figure 6. Distribution of noise tolerance thresholds for STVM parameter in all patients with the median at 490. The $1^{\text {st }}\left(\mathrm{Q}_{1}\right)$ and $3^{\text {rd }}\left(\mathrm{Q}_{3}\right)$ quartiles of the data are also displayed.

\section{Results}

We generated a SNR-controlled noise-added database and derived a model to estimate the SNR levels from the normalized SQI noise measures. The variation in the ECG parameters across each 5-minute recording was used as a measure of noise level. A noise tolerance threshold was statistically derived for each ECG parameter and is listed in Table 2. This means that the parameter values from a recording with SQI noise measure above this threshold are not reliable and should not be used in clinical decision making.

Table 2. Noise tolerance thresholds. Parameter values from a recording with SQI noise measure above this threshold should not be used.

\begin{tabular}{lc}
\hline \hline Parameter & Threshold \\
\hline \hline Heart Rate & 970 \\
PR interval & 400 \\
QRS duration & 640 \\
QT interval & 470 \\
QT $_{\text {c interval }}$ & 450 \\
P-wave axis & 280 \\
QRS axis & 700 \\
T-wave axis & 320 \\
P-wave amplitude & 380 \\
Q-wave amplitude & 800 \\
R-wave amplitude & 410 \\
S-wave amplitude & 530 \\
T-wave amplitude & 360 \\
ST amplitude & 440 \\
ST-VM & 490 \\
\hline
\end{tabular}

\section{Discussion and conclusions}

ECG parameters show different levels of noise tolerance. We statistically derived a threshold for each parameter based on the noise-dependent variations of the ECG parameters. However, these thresholds depend on several factors such as the deviation limit and the deviation criteria we defined. Depending on the application, one may define different settings.

We used a database consisting of 103 patients, however, a larger database will result in a more accurate statistical analysis and a more reliable noise model.

\section{References}

[1] Laguna P, Sörnmo L. The STAFF III ECG database and its significance for methodological development and evaluation. Journal of Electrocardiology. 2014;47(4):408417.

[2] PhysioNet/The MIT-BIH Noise Stress Test Database. https://physionet.org/physiobank/database/nstdb.

[3] Firoozabadi R, Gregg R, Zengo B, Babaeizadeh S. Automatic Real-Time Quality Assessment of a 12-Lead ECG Recording. Computing in Cardiology. 2014;41:589-592.

[4] Online:http://www.usa.philips.com/healthcare/product /HCNOCTN68/dxl-16lead-ecg-algorithm-ecg-algorithm

Address for correspondence.

Reza Firoozabadi

Advanced Algorithm Research Center (AARC), Philips Healthcare reza.firoozabadi@philips.com 